\title{
Energiewende: Wo bleibt das langfristige Denken bei der Offshore-Windenergie?
}

\begin{abstract}
Die Offshore-Windenergie mit Schwerpunkt auf der Nordseeregion und ihren dominierenden Ländern entwickelt sich zuungunsten Deutschlands. Zudem gewinnt Asien in diesem Bereich erhebliche Marktanteile. Offshore-Windenergieanlagen erfordern eine langfristige Planung, wobei die Stilllegung miteinbezogen werden muss. Im nächsten Jahrzehnt ergeben sich zwei große Stilllegungszyklen. Der Stand der Vorbereitungen in den jeweiligen Ländern wird kritisch diskutiert. Offene Fragen zur Anpassung an die Herausforderungen, die sich aus der Stilllegung ergeben, werden hervorgehoben und mögliche Antworten vorgestellt.
\end{abstract}

Aktuell erlebt die kurzfristige Orientierung in der Politik angesichts der Corona-Pandemie einen neuen Höhepunkt, umschrieben als „Auf-Sicht-Fahren“. Während dieses Verhalten bei Unsicherheit kurzfristig Sinn ergibt, ist eine fehlende langfristige Perspektive in anderen Bereichen problematisch. Ein Beispiel hierfür ist die Entwicklung der Offshore-Windenergie, die beispielhaft zeigt, wie erratische Signale der Politik und fehlende Planungssicherheit einen Sektor ausbremsen können. War Deutschland hier früher noch mit prominenter Rolle vertreten, ist eine deutlich divergierende Entwicklung zwischen den Staaten der Nordseeregion zu beobachten. Auch Trends und Szenarien lassen eine Verfestigung dieser Struktur erwarten.

Problematisch wird die stark kurzfristige Orientierung auch hinsichtlich des notwendigen Rückbaus von Offshore-Windenergieanlagen. Der Gedanke an Rückbau ist im ersten Moment kontraintuitiv, dreht sich die Diskussion doch zumeist um den Aufbau neuer Kapazitäten. Dabei darf aber nicht übersehen werden, dass auch Windenergieanlagen eine begrenzte Laufzeit haben und diese besonders auf hoher See durch extreme Witterungseinflüsse und erschwerte Wartungsbedingungen niedriger als bei Anlagen an Land ausfällt. Mit einer geschätzten Laufzeit von etwa 20 Jahren wird das Thema Rückbau von Offshore-Anlagen vor allem im europäischen Nordseeraum in den kommenden Jahren zunehmend an Dringlichkeit gewinnen, wenn mehrere Windparks dieses kritische Alter erreichen. Angesichts dieser planbaren Ent-

(c) Der/die Autor(en) 2020. Open Access: Dieser Artikel wird unter der Creative Commons Namensnennung 4.0 International Lizenz (https:// creativecommons.org/licenses/by/4.0/deed.de) veröffentlicht.

Open Access wird durch die ZBW - Leibniz-Informationszentrum Wirtschaft gefördert.

* Dieser Beitrag ist im Rahmen von „Eco-innovative concepts for the end of offshore wind energy farms lifecycle (DecomTools)" entstanden, https://keep.eu/projects/21430/ (12. August 2020). wicklung ist es umso bemerkenswerter, dass bisher die notwendigen Voraussetzungen hierfür nicht geschaffen worden sind. Deutschland bildet hierbei keine Ausnahme.

\section{Offshore-Windenergie mit guten Aussichten}

Die Transformation des aktuell auf fossilen Energieträgern beruhenden Energiesystems hin zu einem System erneuerbarer Energieerzeugung wird eine der zentralen Herausforderungen der kommenden Jahrzehnte darstellen. Dies gilt besonders vor dem Hintergrund einer global steigenden Energienachfrage und eines gleichzeitig kleiner werdenden Möglichkeitsfensters für diese Energiewende. So hat Deutschland als Ziel ausgegeben, bis $205080 \%$ der Stromnachfrage aus erneuerbaren Quellen decken zu wollen (BMWi, 2019), wobei zunehmend ein höheres Ausbautempo gefordert wird (z. B. Quaschning, 2020).

In diesem Kontext wird einem signifikanten Ausbau der Windenergie als Substitut fossiler Energieträger eine besondere Stellung zugemessen. Hier waren in der Vergangenheit bereits deutliche Reduktionen der Erzeugungskosten zu beobachten: Lag der Preis pro MWh 2015 noch bei 160 Euro, sank dieser 2019 mit 80 Euro auf die Hälfte, wobei ein Preis von 50 Euro pro MWh bis 2030 als realistisch eingeschätzt wird (BWE, 2019). Sowohl Offshore- als auch Onshore-Windkraftanlagen werden daher absehbar wichtige Säulen emissionsarmer Energieerzeugung sein. Dabei weisen Anlagen auf hoher See einen

Mirko Kruse, M.A., ist Junior Researcher am Hamburgischen WeltWirtschaftsInstitut (HWWI). 
höheren Effizienzgrad auf, da hier die Windgeschwindigkeiten höher sind und weniger Verwirbelungen gemessen werden (Belgian Offshore Platform, 2019). Hinzu kommt, dass Offshore-Anlagen von der erneut aufgeflammten Diskussion um Abstandsregelungen zu Wohnbebauung unberührt bleiben und, wie im Fall der deutschen Hochsee-Windparks, vom Ufer aus kaum erkennbar sind, was Proteste von dieser Seite deutlich reduziert (Fachagentur Windenergie an Land, 2020). Allerdings weisen OffshoreAnlagen ebenso spezifische Nachteile auf, darunter der erhöhte Aufwand für Konstruktion und Wartung verglichen mit Anlagen an Land oder die hohe Materialintensität von rund $500 \mathrm{t}$ Stahl und $1.000 \mathrm{t}$ Beton zur Installation von einem MW Leistung (Cohen, 2019; Peters et al., 2005). Nichtsdestotrotz sollen jene den Fokus der folgenden Betrachtungen darstellen.

\section{"Shift to Asia“" und Ambitionen in Europa}

Auf globaler Ebene hat sich die Offshore-Windenergie in den letzten zwei Jahrzehnten zu einer internationalen Industrie entwickelt. Der Großteil der Kapazitätssteigerung entfiel dabei in der Vergangenheit auf Europa, wobei Asien und die USA in den vergangenen Jahren in einen Aufholprozess eingetreten sind: Während die Steigerungsrate in Europa 2018 gegenüber dem Vorjahr bei $16 \%$ lag, hat sich die Kapazität in China innerhalb eines Jahres verdreifacht (IEA, 2020). Bereits 2018 entfielen rund $20 \%$ der globalen Offshore-Windkapazität auf China (im Vergleich zu $28 \%$ in Deutschland und $34 \%$ in Großbritannien) (GWEC, 2019). Angesichts der höheren Wachstumsrate ist zu erwarten, dass China 2020 die globale Führungsrolle bei OffshoreWindenergie übernimmt, wie sie es bereits seit längerem für Onshore-Windenergie innehat (Weber, 2019).

Auch bei den Investitionen ist eine Verschiebung nach Asien zu konstatieren: So entfielen 2018 rund die Hälfte der weltweiten Investitionen in Offshore-Windenergie auf China, das bis 2030 eine Kapazität von $30 \mathrm{GW}$ anstrebt. Auch andere asiatische Länder haben für das kommende Jahrzehnt ambitionierte Ziele ausgegeben, von 5,5 GW (Taiwan) bis hin zu $18 \mathrm{GW}$ (Südkorea) oder sogar $30 \mathrm{GW}$ (Indien) (Cohen, 2019). Zum Vergleich: Die gesamte globale Kapazität für Offshore-Windenergie wurde 2018 noch mit 23GW angegeben (GWEC, 2019). Angesichts der Ausbaupotenziale ist eine Verfestigung dieser Entwicklung und eine weitere Verschiebung in Richtung Asien zu erwarten (Bernthal-Hooker, 2019). Der ursprünglich vorwiegend europäisch dominierte Markt für Offshore-Windenergie hat sich zunehmend ausdifferenziert und internationalisiert (BMWi, 2015).

Nichtsdestotrotz ist der Markt in Europa weit entfernt davon zu stagnieren: In einem Basisszenario erwartet Wind
Europe (2017) bis 2030 eine Verdopplung der Kapazitäten gegenüber 2016. Zwar sind diese Berechnungen im Vergleich eher als optimistisch einzuordnen, angesichts der angestrebten Dekarbonisierung im Energiesektor, wie sie nun auch der European Green Deal in den Fokus nimmt, sind ambitionierte Zielwerte wie dieser aber nicht per se auszuschließen (Europäische Kommission, 2019). Der Weg ist bereits eingeschlagen: Wurden 2016 europaweit noch $10,4 \%$ des Strombedarfs durch Windenergie (onund offshore) erzeugt, sollen bis 2030 mindestens $27 \%$ aus erneuerbaren Quellen kommen (NorthSEE, 2017a; Europäische Kommission, 2018).

\section{Nordseeregion mit europäischer Führungsrolle}

Zusammengenommen waren in Europa Ende 2018105 Offshore-Windfarmen mit 4.543 Turbinen in Betrieb. Dabei konzentrierten sich $98 \%$ der europäischen OffshoreWindkapazität auf nur fünf Länder: Großbritannien (44\%), Deutschland (34\%), Dänemark (7\%), Belgien $(6,4 \%)$ und die Niederlande (6\%) (Wind Europe, 2019). Es ist kein Zufall, dass die fünf größten Erzeuger von Offshore-Windenergie an der Nordsee liegen. Diese bietet besonders gute Voraussetzungen für Windstromerzeugung auf See, resultierend aus relativ niedrigen Wassertiefen, hohen Windgeschwindigkeiten und seltenen außerordentlichen Sturmereignissen (NEP, 2018). Entsprechend befinden sich $62 \%$ der installierten Kapazität zur Offshore-Winderzeugung in Europa in der Nordsee, verglichen mit $15 \%$ in der Irischen See, 14\% in der Ostsee und 9\% im Atlantik (Wind Europe, 2019). Von den $750.000 \mathrm{~km}^{2}$ der Nordsee werden bereits $3.500 \mathrm{~km}^{2}$ zur Stromerzeugung genutzt, wobei eine Steigerung auf über $8.000 \mathrm{~km}^{2}$ bis $2030 \mathrm{er}-$ wartet wird (NorthSEE, 2017b).

Innerhalb der Nordseeanrainerstaaten ist in nahezu allen Ländern ein steigender Trend bei der Kapazität von Offshore-Windenergie über die letzten Jahre festzustellen. Einzelne Länder, wie z.B. die Niederlande, operierten 2016 noch auf relativ niedrigem Niveau (357 MW), konnten dieses aber bis 2018 (957 MW) deutlich steigern. Dies entspricht einer Zunahme um 168,1\%. Auch in Belgien nahm die Kapazität zwischen 2016 (712 MW) und 2018 (1.186 MW) mit 66,6\% stark zu. In Dänemark wurde zwar 2017 altersbedingt ein Windpark vom Netz genommen, zwischen 2016 (1.271 MW) und 2018 (1.701 MW) stieg die installierte Kapazität jedoch trotzdem um 33,8\%. Die hohen Wachstumsraten können zwar teilweise als Aufholprozess interpretiert werden, aber auch Großbritannien, das bei der Offshore-Windkapazität europaweit an der Spitze liegt, konnte zwischen 2016 (5.103 MW) und 2018 (7.525 MW) um $47,5 \%$ zulegen (Kruse, 2019) (vgl. Abbildung 1). Norwegen als weiterer Anrainerstaat betreibt bis auf eine Testanlage mit 2,3 MW keine Offshore-Windanlagen in der Nordsee, 
Abbildung 1

Installierte Leistung aus Offshore-Windenergie im Nordseeraum 2016 bis 2018

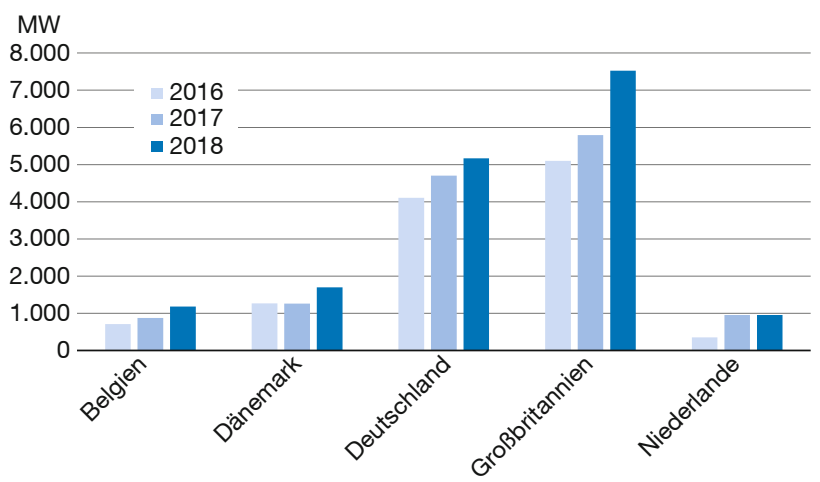

Quelle: Darstellung nach Daten aus Kruse (2019).

während Schweden zwar in der Vergangenheit entsprechende Anlagen vorweisen konnte, diese nach ihrem Abbau aber bisher nicht durch neue ersetzt hat. Beide werden nachfolgend entsprechend nicht betrachtet.

\section{Deutschland wird abgehängt}

Während die anderen Nordseeländer, unabhängig von der bereits installierten Kapazität, deutliche Kapazitätssteigerungen realisierten, macht sich in Deutschland ein verlangsamter Trend bemerkbar: Lag zwischen 2016 (4.108MW) und 2017 (4.705MW) der Kapazitätsanstieg noch in etwa auf dem Niveau Großbritanniens, war 2018 eine deutliche Abschwächung erkennbar. Mit einem Zubau von 9,9\% zwischen 2016 (4.108MW) und 2018 (5.169 MW) lag Deutschland an letzter Stelle der fünf betrachteten Nordseestaaten (Kruse, 2019). Zwar können marktübliche Schwankungen als Teil der Begründung herangezogen werden, hinzu kommt jedoch ein erheblicher Grad von Verunsicherung durch die politische Rahmensetzung. $\mathrm{Zu}$ nennen ist hier beispielsweise die Reform des Erneuerbare-Energien-Gesetzes (EEG) 2017. Hier wurde ein Systemwechsel vom Modell der Einspeisevergütung hin zum Ausschreibungsverfahren vollzogen und gleichzeitig eine Deckelung der Ausbauquoten eingeführt (BMWi, 2017). Bereits bei der Novellierung des EEG war von Fachverbänden davor gewarnt worden, die veränderte politische Rahmensetzung könnte eine deutliche Ausbremsung der Energiewende zur Folge haben (BEE, 2016).

Der Eindruck einer strukturellen Verlangsamung in Deutschland verstärkt sich mit Blick auf die Kapazität, die sich im Bau befindet. So wird in Belgien durch Fertigstellungen von Anlagen in den kommenden Jahren mit 1.076 MW eine Kapazität zusätzlich hinzukommen, die nahezu der Gesamtkapazität des Landes 2018 entspricht
Abbildung 2

Geplante Leistung aus Offshore-Windenergie im Nordseeraum bis 2030

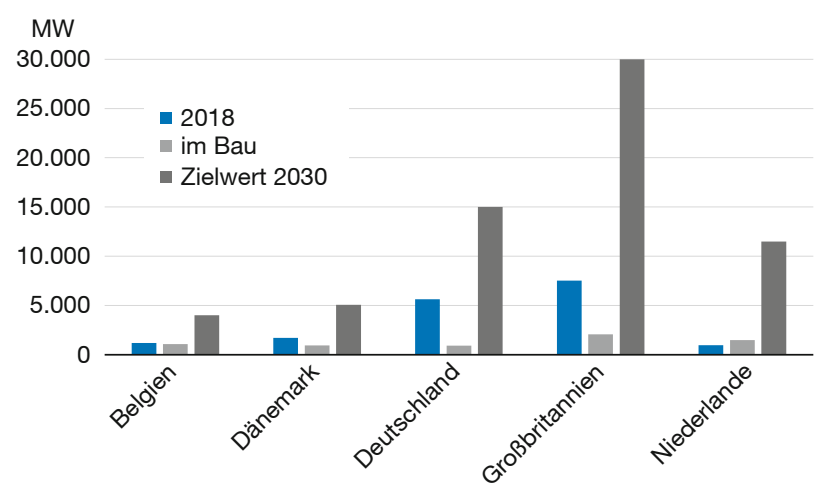

Quelle: Darstellung nach Daten aus Kruse (2019).

(1.186 MW). In den Niederlanden befindet sich sogar mehr Kapazität im Bau (1.483 MW) als 2018 angeschlossen war (957 MW), während Dänemark durch Fertigstellungen die Kapazität von Offshore-Windenergie in der Nordsee um $55,8 \%$ steigert und Großbritannien immerhin noch zusätzliche 27,4\% im Bau hat. Deutschland liegt mit 17,6\% im Bau befindlicher Kapazität, bezogen auf 2018 als Basisjahr, an letzter Stelle. Die Ausbauziele in allen fünf Ländern bis 2030 bewegen sich in einem Korridor von $4 \mathrm{GW}$ (Belgien) bis $30 \mathrm{GW}$ (Großbritannien). Deutschland befindet sich mit $15 \mathrm{GW}$ zwar vor Belgien oder Dänemark (5,1 GW), droht aber von den Niederlanden abgehängt zu werden, die bis 2030 in der Nordsee 11,5 GW aus Windenergie erzeugen wollen (Kruse, 2019) (vgl. Abbildung 2). Während sich die hohen Ziele Großbritanniens aus der Insellage des Landes ergeben, ist für Deutschland zu beachten, dass die Ausbauziele sich auf Nord- und Ostsee zusammen beziehen und in Relation zur Küstenlänge anderer Nordseeländer nicht besonders ambitioniert erscheinen. Eine strukturelle Verschiebung innerhalb dieser Gruppe zeigt sich ebenso mit Blick auf die Neuinvestitionen: Während Großbritannien 2018 mit umgerechnet 5,4 Mrd. Euro unangefochten an der Spitze lag, folgten mit einigem Abstand Belgien (1,8 Mrd. Euro), die Niederlande (1,4 Mrd. Euro) und Dänemark (1,1 Mrd. Euro). Deutschland, beim Bestand noch auf Platz 2 liegend, verliert mit Investitionen von 0,4 Mrd. Euro bereits den Anschluss (Wind Europe, 2019).

\section{Die Ersten werden nicht die Letzten sein}

Einen zusätzlichen Aspekt stellt das Alter der OffshoreWindenergieanlagen dar. Jene Länder in der Nordseeregion, die Pioniere in der Offshore-Windenergie waren, besitzen heute die ältesten Anlagen. So steht die älteste, 1995 errichtete Anlage in der Nordsee (Tunoe Knob) in dä- 


\section{Abbildung 3}

Fertigstellung von Offshore-Windparks in der Nordseeregion

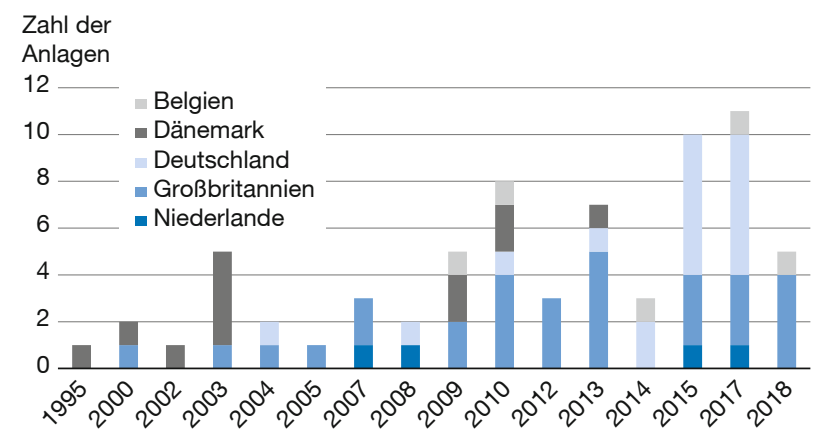

Quelle: Darstellung nach Daten aus Kruse (2019).

nischen Gewässern, wo auch zwei weitere der vier ältesten Anlagen in der Nordsee (Baujahr 2000 und 2002) stehen. Der vierte dieser Windparks steht vor Großbritannien und wurde 2000 gebaut. Am Alter der Anlagen lassen sich zudem die Entwicklungszyklen der Windkraftindustrie nachvollziehen: So wurde die erste deutsche Pilotanlage in der Nordsee 2004 vollendet, während Dänemark und Großbritannien zu diesem Zeitpunkt bereits etablierte Akteure in diesem Bereich waren. Als in Deutschland 2008 eine weitere Pilotanlage in Betrieb genommen wurde und 2010 mit Alpha Ventus schließlich der erste vollwertige Windpark folgte, stiegen die Niederlande 2007 und 2008 mit ihren ersten Anlagen ein. Belgien folgte ein Jahr später. In Deutschland ist der Durchbruch für Offshore-Windenergie in den Jahren ab Mitte des letzten Jahrzehnts zu verorten, als nacheinander 2014 zwei Windparks, 2015 sechs Windparks und 2017 weitere sechs in Betrieb genommen wurden (vgl. Abbildung 3).

Zu beachten ist, dass die Durchschnittsgröße von Offshore-Windparks im Zeitverlauf deutlich gestiegen ist. So bestand der erste Offshore-Windpark in der Nordsee 1995 noch aus zehn Turbinen, während die durchschnittliche Turbinenzahl 2018 bei fast 70 lag. Zwischenzeitlich lag der Durchschnitt sogar, wegen vier großer britischer Windparks, bei 133 Turbinen jährlich (2010). Allgemein ist ab 2009 ein starker Anstieg der jährlich neu angeschlossenen Turbinen festzustellen, wobei die bisherigen Rekordjahre 2010 (1.067 Turbinen) und 2015 (797 Turbinen) waren.

\section{Rückbau rückt auf die Agenda}

Während die Diskussion über viele Jahre von einem Ausbau der Offshore-Windkraftanlagen geprägt war, gerät nun zunehmend eine neue Herausforderung in den Blick: die älteren Anlagen zurückzubauen oder durch neue zu ersetzen. Während Windkraftanlagen an Land etwa 20 bis

\section{Abbildung 4}

Erwartete Zahl zurückzubauender OffshoreWindenergieanlagen in der Nordseeregion ${ }^{1}$

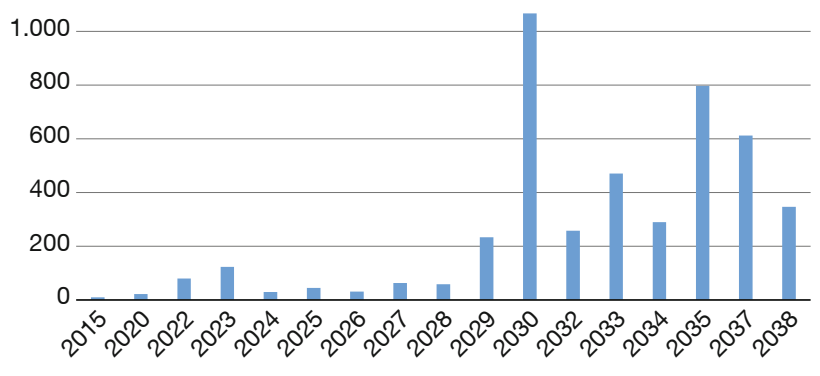

${ }^{1}$ Angenommen wurde eine Laufzeit von 20 Jahren.

Quelle: Darstellung nach Daten aus Kruse (2019).

25 Jahre betrieben werden, wird für Anlagen im OffshoreBereich eher eine Laufzeit von 20 Jahren angenommen, die sich aus zeitlich begrenzten Betriebsgenehmigungen, extremen Witterungseinflüssen und erschwerten Wartungsbedingungen ergibt. Hinzu kommt, dass die ersten Offshore-Anlagen technisch noch nicht auf dem heutigen Stand waren und entsprechend einen höheren Verschleißgrad aufweisen.

Im europäischen Nordseeraum als Pilotregion der Offshore-Windenergie sind in Bezug auf den Rückbau von Anlagen zwei größere Zyklen absehbar: Der erste Zyklus setzt aktuell ein und streckt sich bis 2023 , wenn in der Nordseeregion 22 (2020), 80 (2022) und 123 (2023) Turbinen jährlich zurückzubauen sind. Dieses Marktvolumen stellt eine Schwierigkeit dar, da die Zahlen zurückzubauender Anlagen vorläufig noch zu niedrig sind, um ein hohes Marktvolumen zu begründen. Teile der Wertschöpfungskette, wie z. B. der Recyclingbereich, profitieren indes bereits von anderen Teilmärkten wie dem Rückbau von Onshore-Anlagen. Der zweite Zyklus setzt spätestens ab 2029 ein, wenn ein Großteil der heutigen Anlagen nach und nach ans Ende ihrer Laufzeit gelangt. Jährliche Rückbauzahlen von 234 (2029) und 1.067 (2030) Turbinen zeigen, dass hier ein signifikantes Volumen zu erwarten ist (vgl. Abbildung4). Während der erste Zyklus noch als Testfeld verstanden werden kann, die effizientesten Technologien $z u$ identifizieren und die entsprechenden Infrastrukturen zu schaffen, müssen diese zu Beginn des zweiten Zyklus bereits etabliert sein, um die hohen Rückbauzahlen bewältigen zu können.

\section{Mangelnde Erfahrung beim Rückbau offshore}

Während in den kommenden Jahren die Frage des Rückbaus von Offshore-Windkraftanlagen zunehmend lauter gestellt werden wird, halten sich die bisherigen Erfahrun- 
gen in Grenzen. Zwar sind Rückbauprozesse an Land bekannt und in zahlreichen Projekten erprobt, jedoch fehlt dieses Erfahrungsniveau im Offshore-Bereich. Beispielprojekte wie der Rückbau von Offshore-Windparks wie Vindeby/Dänemark (errichtet 1991, zurückgebaut 2017), Utgrunden/Schweden (errichtet 2000, zurückgebaut 2018), Yttre Stengrund/Schweden (errichtet 2001, zurückgebaut 2016) oder Blyth/Großbritannien (errichtet 2000, zurückgebaut 2019) sind rar gesät. Hinzu kommen einzelne zurückgebaute Testturbinen in unmittelbarer Küstennähe. Die Laufzeiten dieser Anlagen weisen dabei eine hohe Varianz auf und zeigen, dass ein Rückbau auch schon vor Ablauf der angenommenen 20 Jahre eine Option werden kann. Die tatsächliche Laufzeit wird von unterschiedlichen Faktoren wie dem mechanischen Verschleiß, Reparaturkosten oder einer möglichen Nachnutzung der Fläche durch größere und effizientere Anlagen beeinflusst.

Angesichts der überschaubaren Zahl von Beispielprojekten besteht auch bei den zu erwartenden Kosten ein hohes $\mathrm{Maß}$ an Unsicherheit. Erste Kalkulationen aus Großbritannien rechneten 2010 mit Rückbaukosten in Höhe von 40.000 Pfund pro MW, was einem Anteil von 2,5\% der gesamten Projektkosten entsprochen hätte (Climate Chance Capital, 2010). Diese Berechnungen sind mittlerweile zurückgezogen und durch neue Kalkulationen ersetzt worden, die deutlich höhere Kosten von 100.000 Pfund bis 300.000 Pfund pro MW nahelegen (Topham und McMillan, 2017).

Für die Rückbauplanungen wirkt sich positiv aus, dass die älteren Anlagen tendenziell kleiner und in flacheren Gewässern liegen als moderne Anlagen. So ist im Zeitverlauf eine deutliche Zunahme sowohl der Anlagengröße (Höhe, Rotordurchmesser), der Wassertiefen oder der Leistung festzustellen. Befinden sich die Offshore-Windanlagen von 1995 in Tiefen zwischen 0,8 $\mathrm{m}$ und $5 \mathrm{~m}$, lag der Korridor 2010 zwischen $4 \mathrm{~m}$ und 27,5 m und stieg 2018 auf $6 \mathrm{~m}$ bis $36 \mathrm{~m}$ an. Allerdings war in den Pionierjahren der Offshore-Windenergie die Zahl der Produzenten deutlich höher, ehe sich die heute dominierenden Firmen (vor allem Siemens und Vestas) durchsetzen konnten. Auch die Vielfalt getesteter Fundamentvarianten war in früheren Jahren deutlich größer. Zusammengenommen hat dies zur Folge, dass es für den Rückbau der älteren Anlagen keine One-size-fits-all-Lösung geben kann.

\section{Konkrete Herausforderungen für einen nachhaltigen} Rückbau

In Teilen kann auf Erfahrungen aus der Öl- und Gasindustrie zurückgegriffen werden, die im Offshore-Bereich schon länger aktiv ist und entsprechende Kompetenzen beim Rückbau besitzt. Dabei werden jedoch an die Windener- giebranche besonders hohe Maßstäbe angelegt, was die Nachhaltigkeit ihrer Anlagen betrifft. Dementsprechend ist ein effizient und nachhaltig organisierter Rückbauprozess Voraussetzung dafür, den nachhaltigen Anspruch über die gesamte Wertschöpfungskette einer Windkraftanlage abbilden zu können. Fallstricke gibt es beim Rückbau genug: angefangen bei der effizienten Einsatzplanung der dieselbetriebenen Spezialschiffe bis hin zur Frage, wie viele Komponenten wiederverwertet und wie der Anteil nicht recycelbaren Materials möglichst niedrig gehalten werden kann. Hinzu kommen praktische Herausforderungen, wie z. B. die Vorhaltung entsprechender Kapazitäten zur Lagerung und Weiterverarbeitung von Bauteilen in Häfen oder die Verfügbarkeit von Fachpersonal.

Obwohl am Rückbau der älteren Anlagen kein Weg vorbeiführt, da die freiwerdende Fläche benötigt wird und auch die Rückbauvolumina sich abschätzen lassen, ist die politische Regulierung auf die Herausforderung nicht vorbereitet. So sehen zwar die Betriebsgenehmigungen eines Windparks den Rückbau der Anlagen nach Ende der Laufzeit vor, unklar ist jedoch, wie weitreichend diese Vorschrift auszulegen ist. So bestehen in manchen Nordseestaaten keine konkreten Vorgaben und wo solche festgelegt wurden, unterscheiden sie sich zwischen den einzelnen Ländern. Ob unter „Rückbau“ also eine komplette Entfernung des Fundaments und aller Kabel zu verstehen ist, oder ob es eine abgeschwächte Variante wie in Deutschland zu Land gibt, wo das Fundament $1,5 \mathrm{~m}$ unter der Oberfläche abgetragen und darunter an Ort und Stelle gelassen wird, ist nicht einheitlich geregelt.

Ein weiteres Problem stellt das Recycling der Flügel der Anlagen dar, die aus Verbundmaterialien gefertigt werden. Aufgrund ihrer chemischen Eigenschaften sind diese mit herkömmlichen Verfahren nicht wieder sortenrein zu trennen und daher kaum zu recyceln (Liu und Barlow, 2017). Bisherige Verfahren sehen höchstens eine Verwendung als Füllmaterial vor, wobei je nach Land auch die Deponierung von Flügelteilen gängige Praxis ist. Erschwert wird ein adäquates Recycling dadurch, dass die Dokumentation verwendeter Baustoffe, Farben oder Lacke oftmals unvollständig ist. Im Beispiel des Rückbaus des Windparks Vindeby war außerdem das Fundament deutlich massiver als angenommen, was wiederum auf eine unzureichende Dokumentation verweist, die den Planungsprozess erheblichen Unsicherheiten aussetzt (Topham und McMillan, 2017).

Kaum diskutiert wurden bisher zudem biologische Aspekte, die den Rückbauprozess erschweren können. Hierzu zählt z. B. zusätzliches Gewicht der Fundamente und Komponenten durch Bewuchs mit Muscheln, Algen und anderen marinen Lebensformen, was in die Planun- 
gen mit einzubeziehen ist. Hinzu kommen marine Lebewesen, die ihren Lebensraum an den Anlagen gefunden haben. So mussten beispielsweise bestimmte Korallenarten (z. B. Lophelia pertusa), die unter Schutz stehen, bei Rückbauprojekten von Öl- und Gas-Infrastrukturen umgesiedelt werden (Alvarez et al., 2012).

\section{Fazit - Chancen für Europa}

Die Herausforderungen des Rückbaus von OffshoreWindanlagen werden in Europa zuerst akut, bleiben aber nicht hierauf beschränkt. Mit einem entsprechenden Zeitverzug werden auch alle anderen Weltregionen, die in der Offshore-Windenergie aktiv sind oder dies planen, vor den gleichen Fragen stehen. Europa hat an dieser Stelle die Chance, den Standard für Rückbauprozesse zu setzen, der künftig weltweit Anwendung findet. Von einem effizienten Gesamtkonzept würden langfristig Unternehmen und Forschungsinstitute profitieren, die ihr akkumuliertes Know-how weltweit anbieten können. Der Prozess würde ebenso die Nachhaltigkeitsstrategie im Sinne des European Green Deal unterstützen. Notwendig wäre allerdings, die kurzfristige Perspektive aufzugeben und die langfristige Entwicklungsperspektive des Marktes in den Blick zu nehmen. Wie Unsicherheit einen funktionierenden Markt schädigen kann, zeigt das Beispiel der deutschen Offshore-Windenergie eindrücklich.

\section{Literatur}

Alvarez, M. C., E. Bell und M. Elliott (2012), Causes and Consequences of Odours from Marine Growth Organisms, https://oilandgasuk.co.uk/ wp-content/uploads/2019/07/The-Management-of-Marine-Growthduring-Decommissioning.pdf (19. August 2020).

Belgian Offshore Platform (2019), FAQ - Economy, https://www.belgianoffshoreplatform.be/en/faq (14. April 2020).

Bernthal-Hooker, J. (2019), Vineyard moves three turbines, https:// www.4coffshore.com/news/newsltem.aspx?nid=13850 (19. August 2020).

BMWi (Bundesministerium für Wirtschaft und Energie) (2015), The energy transition - a great piece of work. Offshore wind energy - An overview of activities in Germany.

BMWi (2017), Die nächste Phase der Energiewende: Das EEG 2017, https://www.bmwi.de/Redaktion/DE/Artikel/Energie/eeg-2017-start-indie-naechste-phase-der-energiewende.html (19. August 2020).
BMWi (2019), Technologien, Windenergie auf See, Ziele, https://www. erneuerbare-energien.de/EE/Navigation/DE/Technologien/Windenergie-auf-See/Ziele/ziele.html (19. August 2020).

BEE (Bundesverband Erneuerbare Energie) (2016), EEG-Reform 2016 schneidet hart ins Herz der Energiewende, https://www.bee-ev.de/ presse/mitteilungen/detailansicht/eeg-reform-2016-schneidet-hartins-herz-der-energiewende (19. August 2020).

BWE (Bundesverband WindEnergie) (2019), Zahlen und Fakten, https:// www.wind-energie.de/themen/zahlen-und-fakten (14. April 2020).

Climate Change Capital (2010), Offshore Renewable Energy Installation Decommissioning Study, Final Report (withdrawn), https://assets.publishing.service.gov.uk/government/uploads/system/uploads/attachment_data/file/608023/900-offshore-renewable-installation-decom. pdf (19. August 2020).

Cohen, A. (2019), As Global Energy Demand Grows, So Does Appetite for Offshore Wind, https://www.forbes.com/sites/arielcohen/2019/03/26/as-global-energy-demands-grows-so-does-appetite-for-offshore-wind (19. August 2020).

Europäische Kommission (2018), Renewable Energy - Moving towards a low carbon economy, https://ec.europa.eu/energy/topics/renewableenergy_en (19. August 2020).

Europäische Kommission (2019), The European Green Deal, COM(2019) 640 final, https://ec.europa.eu/info/sites/info/files/european-greendeal-communication_en.pdf (19. August 2020).

Fachagentur Windenergie an Land (2020), Überblick zu den Abstandsempfehlungen zur Ausweisung von Windenergiegebieten in den Bundesländern, https://www.fachagentur-windenergie.de/fileadmin/files/ PlanungGenehmigung/FA_Wind_Abstandsempfehlungen_Laender. pdf (19. August 2020).

GWEC (Global Wind Energy Council) (2019), Global Wind Report 2018 , April.

IEA (International Energy Agency) (2020), Offshore Wind - Tracking Clean Energy Progress, https://www.iea.org/reports/offshore-wind (10. September 2020)

Kruse, M. (2019), Market Analysis Decom Tools 2019, https://northsearegion.eu/media/11753/market-analysis_decomtools.pdf (9. April 2020).

Liu, P. und C. Y. Barlow (2017), Wind turbine blasé waste in 2050, Waste Management, 62(2017), 229-240.

NEP (Norwegian Energy Partners) (2018), Annual global offshore wind market report 2019.

NorthSEE (2017a), Offshore renewable energy development - Offshore Wind, https://northsearegion.eu/northsee/e-energy/offshore-renewable-energy-developments-offshore-wind/ (19. August 2020).

NorthSEE (2017b), Analysis of future outlook, https://northsearegion.eu/ northsee/e-energy/analysis-of-future-outlook/ (19. August 2020).

Peters, P. F., H. Zhao und R. Petroski (2005), Metal and Concrete Inputs for Several Nuclear Power Plants, http://fhr.nuc.berkeley.edu/wp-content/uploads/2014/10/05-001-A_Material_input.pdf (20. August 2020).

Quaschning, V. (2020), Erneuerbare Energien und Klimaschutz: Hintergründe - Techniken und Planung - Ökonomie und Ökologie - Energiewende, Carl Hanser.

Topham, E. und D. McMillan (2017), Sustainable decommissioning of an offshore wind farm, Renewable Energy, 102(2017), 470-480.

Weber, T. (2019), China übernimmt Führung der Offshore-Windkraft-Länder, https://www.erneuerbareenergien.de/china-uebernimmt-fuehrung-der-offshore-windkraft-laender (19. August 2020).

Wind Europe (2017), Wind energy in Europe: Scenarios for 2030.

Wind Europe (2019), Offshore Wind in Europe - Key trends and statistics 2018.

Title: Energy Transition: Where is the Long-Term Thinking on Offshore Wind Energy?

Abstract: The paper at hand presents the development of offshore wind energy with a focus on the North Sea Region (NSR) and its dominant countries. The article analyses not only the shift to Asia in terms of offshore energy but also a structural shift inside the NSR that is disadvantageous to Germany. As an example of longterm planning within the market, the article quantitately examines the new challenge of decommissioning offshore wind installations embedded in two major cycles arising in the next decade. Moreover, the level of preparation in the respective countries is critically discussed. Open questions about adapting to the challenges arising from decommissioning are highlighted and potential answers are presented.

JEL Classification: Q01, Q42, Q48 